\title{
Moiré beatings in graphene on $\mathrm{Ru}(0001)$
}

\author{
Marcella Iannuzzi, ${ }_{1}^{1}$ Irakli Kalichava, ${ }^{2}$ Haifeng Ma, ${ }^{3}$ Steven J. Leake, ${ }^{2}$ Haitao Zhou, ${ }^{4}$ Geng Li, ${ }^{4}$ Yi Zhang, ${ }^{4}$ \\ Oliver Bunk, ${ }^{2}$ Hongjun Gao, ${ }^{4}$ Jürg Hutter, ${ }^{1}$ Philip R. Willmott, ${ }^{2}$ and Thomas Greber ${ }^{3, *}$ \\ ${ }^{1}$ Physikalisch-Chemisches Institut, Universität Zürich, Winterthurerstrasse 190, 8057 Zürich, Switzerland \\ ${ }^{2}$ Paul Scherrer Institut, 5232 Villigen, Switzerland \\ ${ }^{3}$ Physik-Institut, Universität Zürich, Winterthurerstrasse 190, 8057 Zürich, Switzerland \\ ${ }^{4}$ Institute of Physics, Chinese Academy of Sciences, Beijing, 100190, China
}

(Received 24 February 2012; revised manuscript received 29 August 2013; published 27 September 2013)

\begin{abstract}
The moiré superstructure of a single layer of carbon on ruthenium, where $25 \times 25$ graphene honeycombs sit on $23 \times 23$ substrate unit cells, is determined from first principles. The density functional theory (DFT) calculations predict two kinds of structural units, $\Omega$ and $Y$, in the supercell, which are identified as moiré beatings or moirons. The related topographic bucklings, or "hills," have distinct carbon conformations and a height of $1.16 \AA$. The different moirons are observed with scanning tunneling microscopy (STM), and surface x-ray diffraction (SXRD) also discriminates the two. This connects ab initio DFT calculations with STM and SXRD experiments in unit cells containing more than 4000 atoms.
\end{abstract}

DOI: 10.1103/PhysRevB.88.125433

PACS number(s): 61.48.Gh, 68.35.Gy, 68.37.Ef, 68.65.-k

The understanding and use of materials relies on the knowledge of their detailed atomic structure. For single layers of graphene, the main structural parameters are registry with and distance to the substrate, which have a decisive influence on the electronic properties ${ }^{1-4}$ and the functionality. ${ }^{5-8}$ Such systems are commonly called moiré superstructures. The alignment and registry depend on the lock-in of the carbon atoms to the substrate atoms, which is mediated via the sitedependent bonding of the carbon $p_{z}$ orbitals. Since the lock-in energy ${ }^{9}$ is roughly proportional to the adsorption energy, other substrates $^{1}$ and in particular copper ${ }^{1,10}$ are more prone to multiple, rotated moiré structure formation, as compared to the present case of ruthenium. Graphene on ruthenium is a prototype system for the case of graphene with strong bonds to the substrate. ${ }^{11-13}$ It forms a surprisingly large unit cell with $23 \times 23 \mathrm{Ru}$ units on top of which $25 \times 25$ graphene units are accommodated, as determined by surface $\mathrm{x}$-ray diffraction (SXRD). ${ }^{14}$ The 25-on-23 graphene-on-ruthenium $(\mathrm{g} / \mathrm{Ru})$ structure contains four translationally and structurally inequivalent "hills" or moirons, ${ }^{15}$ instead of only one, as would be the case in, for example, a 12-on-11 structure. A "moiron" is a structural unit with one moire beating period, or "point" between the substrate lattice and the overlayer. ${ }^{15}$ If there is more than one moiré beating such as in the 25-on-23 structure the number the individual moirons may contain fractional numbers of atoms, and therefore a precise description must involve the full unit cell. To date, all ab initio models ${ }^{16-19}$ have treated superstructures with only one moiron.

In this paper, the nonrotated 25-on-23 coincidence lattice, abbreviated by $\left(\begin{array}{l}25 \\ 23\end{array}\right)$, is calculated. We performed DFT optimizations up to a depth of six $\mathrm{Ru}$ layers, plus one carbon overlayer (that is, 4424 atoms in total). ${ }^{20}$ Importantly, four different moirons emerge from the DFT calculations. The peaks of the four hills have the same height. Three of these can be mapped on each other through $120^{\circ}$ rotations and are in this sense (though not translationally) equivalent. The fourth, however, has its own distinct structure. This theoretical prediction turns out to be consistent with atomically resolved large-scale scanning tunneling microscopy images and with surface $\mathrm{x}$-ray diffraction data. ${ }^{21}$ The theoretical methods and experimental details are described in the Supplemental Material. $^{22}$

Previous DFT calculations reported the $\left(\begin{array}{c}12 \\ 11\end{array}\right)$ superstructure to be slightly more stable than the $\left(\begin{array}{l}11 \\ 10\end{array}\right)$ and the $\left(\begin{array}{l}13 \\ 12\end{array}\right)$ structures. ${ }^{17}$ The electronic structure of all three indicates a common hybridization between the $\pi$ orbitals of graphene and the $d$ band of the metal wherever the registry favors good contact between $\mathrm{C}$ and $\mathrm{Ru}$ atoms, i.e., when $\mathrm{C}$ pairs occupy (top, fcc), (top, hcp), or bridge sites. ${ }^{18}$ For these registries, the overlayer is close to the metal (about $2 \AA$ ) and the C-C bonds are stretched. In contrast, where the registry is (fcc, hcp), the interaction to the substrate is given by dispersion forces that permit out-of-plane strain, which leads to the formation of protrusions, or "hills."

The $\left(\begin{array}{l}25 \\ 23\end{array}\right)$ model has four regions with (fcc, hcp) registry and is thus expected to form four protrusions. ${ }^{15}$ However, a $\left(\begin{array}{l}25 \\ 23\end{array}\right)$ system obviously cannot be reduced to a $2 \times 2$ replication array of a $\left(\begin{array}{c}12 \\ 11\end{array}\right)$ model. Here, we identify specific features of this superstructure. The DFT results predict a reconstruction, with four graphene protrusions with a corrugation of $1.16 \AA$ and an average adsorption energy of $0.24 \mathrm{eV}$ per $\mathrm{C}$ atom. These four moirons are inequivalent, however, as shown in Figs. 1(a) and 1(b). Two types-labeled $Y$ and $\Omega$-are distinguished. The $Y$ moiron, or hill, is threefold symmetric. It has a central peak $\mathrm{C}$ atom on an hcp site with its three nearest neighbors on fcc sites. A very similar $Y$ hill is also obtained for a $\left(\begin{array}{l}12 \\ 11\end{array}\right)$ model, for which the corrugation amplitude is only slightly larger (see the table in the Supplemental Material ${ }^{22}$ ). The $\Omega$ hills feature a carbon six-ring centered close to the top of a $\mathrm{Ru}$ atom, for which the $\mathrm{C}$ atoms occupy fcc and hcp sites. $\Omega$ hills are also obtained from the optimization of a $\left(\begin{array}{l}13 \\ 12\end{array}\right)$ model, for which the corrugation is slightly smaller, since the initial stretch of the graphene lattice is larger. Importantly, the $\mathrm{Ru}$ is also corrugated [see Fig. 1(c)]. The varying interaction between the graphene layer and the ruthenium leaves characteristic $Y$ and $\Omega$ "footprints" in the substrate, which increases the SXRD sensitivity for the discrimination between $Y$ and $\Omega$ hills. 

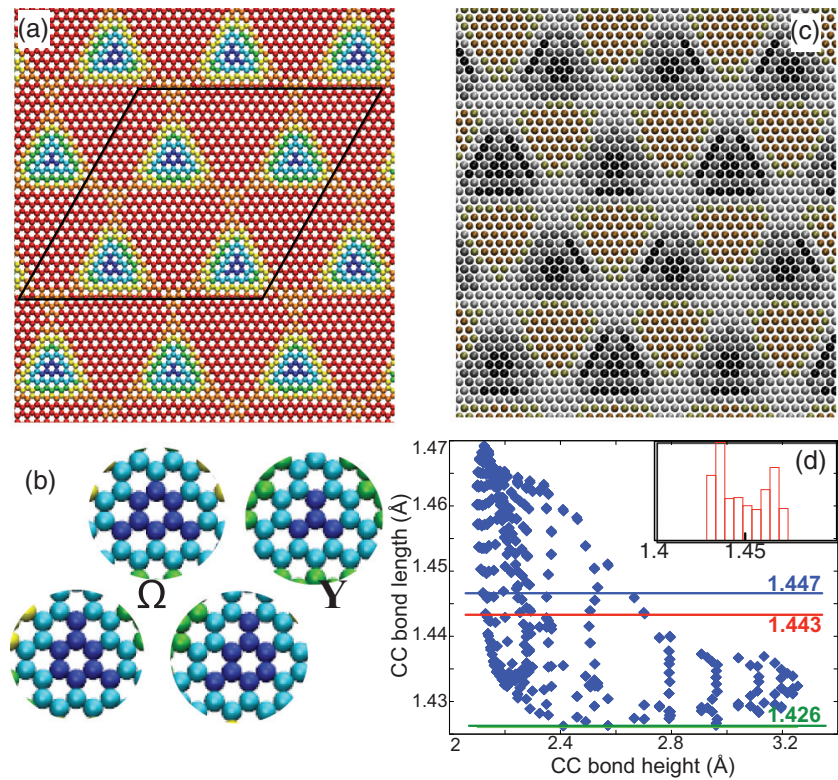

FIG. 1. (Color online) Theoretical results of the arrangement of the $25 \times 25$ carbon atom pairs on top of the $23 \times 23 \mathrm{Ru}$ substrate. (a) Color map of the $\mathrm{C}$ overlayer. The color indicates the height of the $\mathrm{C}$ atoms over $\mathrm{Ru}$ (projected height above the average of the topmost $\mathrm{Ru}$ layer) from about $3.25 \AA$ (blue) to $2.09 \AA$ (red). (b) Details of the four moirons in the unit cell of the superstructure. The dashed lines run along carbon chains that connect the hills. (c) Color map of the first $\mathrm{Ru}$ layer, where the colors indicate the deviation from the average height (set to zero), from $+0.07 \AA$ (black) to $-0.1 \AA$ (brown). (d) $\mathrm{C}-\mathrm{C}$ bond length distribution versus $\mathrm{C}-\mathrm{C}$ bond height. The red horizontal line indicates the initial value (i.e., a flat graphene layer, stretched by $1 \%$ due to heteroepitaxial strain), the blue line is the average value after the optimization, the green line corresponds to the equilibrium value for freestanding graphene (within our model). The inset is a histogram of the bond-length distribution.

The substrate peak-to-peak corrugation decays progressively from $0.17 \AA$ in the topmost Ru layer to $0.03 \AA$ in the fifth layer. The propagation of the corrugation into the Ru slab is in very good agreement with the exponential decay length of the peak-to-peak amplitude deduced from earlier SXRD data analysis. ${ }^{21}$ On those sites where the interaction between graphene and $\mathrm{Ru}$ is strong, the lock-in energy compensates for the graphene lattice strain and the C-C bond length is elongated. The electron density of states (DOS) on these C atoms shows that the bonding states of the $p_{z}$ projection are shifted to lower energies due to hybridization and charge transfer. ${ }^{22}$ Tensile strain and charge transfer into the carbon $p_{z}$ antibonding orbitals expands the graphene lattice. This explains why the $\left(\begin{array}{l}11 \\ 10\end{array}\right)$, which is the commensurate one-moiron structure with the lowest strain energy for flat graphene on flat $\mathrm{Ru},{ }^{13,19}$ is not realized by nature. As soon as the registry with the substrate is unfavorable for bonding, no lattice strain is obtained, and the C-C bond length approaches that of freestanding graphene. The $p_{z}$ DOS on the $Y$ and $\Omega$ hills are very similar, ${ }^{22}$ which is an indication that an exploitation of the differences, like a selective decoration of the $\Omega$ hills is challenging. We do, e.g., find by DFT that lithium atoms adsorb on top of the hills, on hollow sites in the topmost (a)

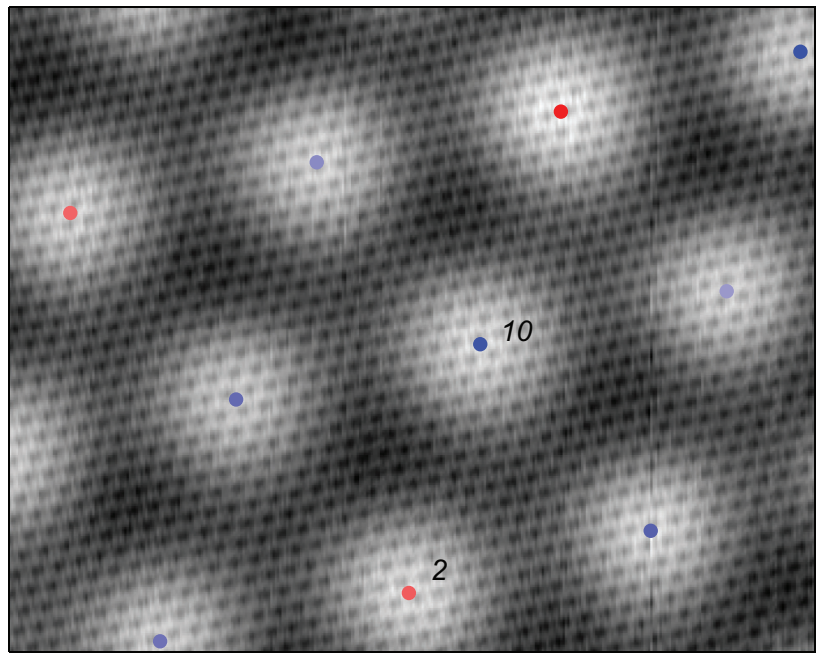

(b)
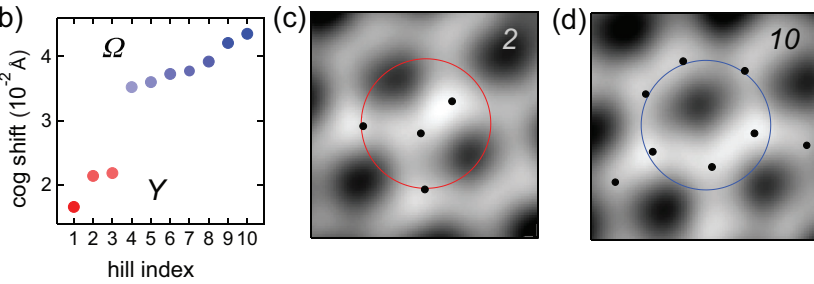

FIG. 2. (Color online) Scanning tunneling microscopy data. (a) Graphene monolayer on $\operatorname{Ru}(0001)\left(98 \times 94 \AA^{2}, V_{t}=-1 \mathrm{~V}\right.$ and $I_{t}=$ $0.1 \mathrm{nA}$, raw data after drift correction, $10^{5}$ pixels). The apparent corrugation is $\approx 0.6 \AA$ and the average height is set to zero. The center positions of the hills are marked blue and red, which indicate large and small shifts of the center of gravity (COG). The COGs are determined in $3.4-\AA$ disks on the centers of the hills, and their shifts are the deviations from those centers. (b) Shifts of the COG. Two distinct groups $Y$ and $\Omega$ can be distinguished. (c) and (d) Smoothed zoom-ins. The red and the blue rings are the perimeters of the disks used for the COG determinations. (c) Hill 2: Quasitetrahedral $Y$ arrangement of the four topmost carbon atoms (dots). (d) Hill 10: Omega arrangement $\Omega$ of the eight topmost carbon atoms (dots).

carbon honeycomb, and that they have small adsorption energy differences in the $\mathrm{meV}$ range. The modulation of the $\mathrm{C}-\mathrm{C}$ bond length with height over the substrate is displayed in Fig. 1(d). The histogram in the inset shows the corresponding bimodal bond-length distribution.

The validity of the $\left(\begin{array}{c}25 \\ 23\end{array}\right)$ model containing two different moiron species has been called into question because of studies indicating a rotation of the carbon lattice away from the substrate high-symmetry directions. ${ }^{19,23,24}$ Using scanning tunneling microscopy (STM), a rotation of $4.5 \pm 0.5^{\circ}$ of the carbon lattice with respect to the Ru substrate was found, ${ }^{23}$ and it was proposed ${ }^{19,23}$ that this is the reason for the $23 \times$ 23 unit cell. Indeed, on the same sample, our STM data also revealed regions where the carbon chains are rotated by $5^{\circ}$ with respect to the centers of the hills. Importantly, however, there were regions where no rotation is observed, as in the case for the $\left(\begin{array}{c}25 \\ 23\end{array}\right)$ DFT model. Figure 2 shows the atomically resolved STM data from the nonrotated $g / \operatorname{Ru}(0001)$ structure, i.e., where the hills are arranged parallel to the carbon chains, as for the results in Fig. 1. Fourier transforms of cuts across the hills 
are compatible with a period of $12 \pm 0.6$ honeycombs between two protrusions, in line with four hills with equal height within the $\left(\begin{array}{l}25 \\ 25\end{array}\right)$ supercell. A closer inspection of the termination of the hills also agrees excellently with the $Y$ and $\Omega$ hills which emerge from the DFT calculations. The center positions of the hills are determined by fitting two-dimensional Gaussians to the STM topographs. Subsequently, the center of gravity of the height within a disk with a radius of $1.7 \AA$ was calculated. Theory predicts no shift of the center of gravity for the $Y$ hills but a significant shift with respect to the $\Omega$ hills [see Fig. 1(b)]. Figures 2(a) and 2(b) show that STM resolves two groups of hills with and without shift, in a ratio which is entirely consistent both with the predicted 3:1 stoichiometry and the corresponding spatial arrangement in the supercell. In Figs. 2(c) and 2(d) zoom-ins for one $Y$ and one $\Omega$ hill are shown.

SXRD was performed at the Materials Science beamline of the Swiss Light Source using 1-Å radiation with improved data acquisition in grazing incidence using a pixel detector. ${ }^{25}$ Four superstructure rods (SSRs) were recorded and then used to fit the DFT data using the genetic algorithm GENX. ${ }^{26}$ By fitting the calculated diffraction intensities from the coordinates of the DFT structure with one graphene and six Ru layers to the SXRD data using a scaling factor and one Debye-Waller factor results in an R-factor of $12.8 \% .{ }^{22}$ It marginally improves to $12.6 \%$ if the artifactual contraction of the interlayer distance of the bottom Ru-layer relaxation due to the vacuum on the bare Ru side of the DFT slab is reset to the bulk Ru-interlayer distance. In both cases, however, it is significantly better than that obtained from the best previous model, which predicted a chiral twist motif of the graphene hills ${ }^{21}(R=18.6 \%$, if the present data set is used). The previous SXRD evaluation ${ }^{21}$ was based on an ansatz in which even Fourier components up to the fourth order were considered for the description of the strain, and the shortest carbon-Ru distance was frozen to values taken from theory. ${ }^{16}$ Introducing chirality decreased the R-factor and strain (Keating) energy compared to a model where $p 3 m 1$ mirror symmetry was preserved. However, the Keating energy for the present $\left(\begin{array}{c}25 \\ 23\end{array}\right)$ model is still lower, decreasing from 9.3 to $6.5 \mathrm{eV}$.

$\mathrm{X}$-ray diffraction is sensitive to the electron density-it is thus at first sight surprising that it is able to clarify the positions of the 7500 carbon electrons with respect to the approximately 3 times more electrons per Ru layer. If we use the superstructure rods for analysis, we reduce the contribution of the Ru substrate significantly. In the following, we show that the SSR data allow us to evaluate the DFT structures, where the straining of the $\mathrm{Ru}$, the $\mathrm{C}-\mathrm{Ru}$ distance, and the graphene corrugation are inferred. In order to demonstrate the ability of SXRD to distinguish between $Y$ and $\Omega$ hills, we compared calculated diffraction intensities from the coordinates of $\left(\begin{array}{l}11 \\ 10\end{array}\right)$, $\left(\begin{array}{c}12 \\ 11\end{array}\right),\left(\begin{array}{c}25 \\ 23\end{array}\right)$, and $\left(\begin{array}{c}13 \\ 12\end{array}\right)$ DFT models, each with four Ru-substrate layers [see Fig. 3(a) and table in Ref. 22]. For this purpose the corresponding SSRs were compared. ${ }^{22}$ The two smallest superstructures favor $Y$ hills, while the $\left(\begin{array}{c}13 \\ 12\end{array}\right)$ consists of an $\Omega$ hill. Figure 3(a) shows the SXRD R-factors for the four structures versus the mismatch of the graphene with respect to the $\mathrm{Ru}$ substrate. The $\left(\begin{array}{c}25 \\ 23\end{array}\right)$ structure has the best $\mathrm{R}$-factor, and the sequence is in line with the $1: 3$ weight of $Y$ hills
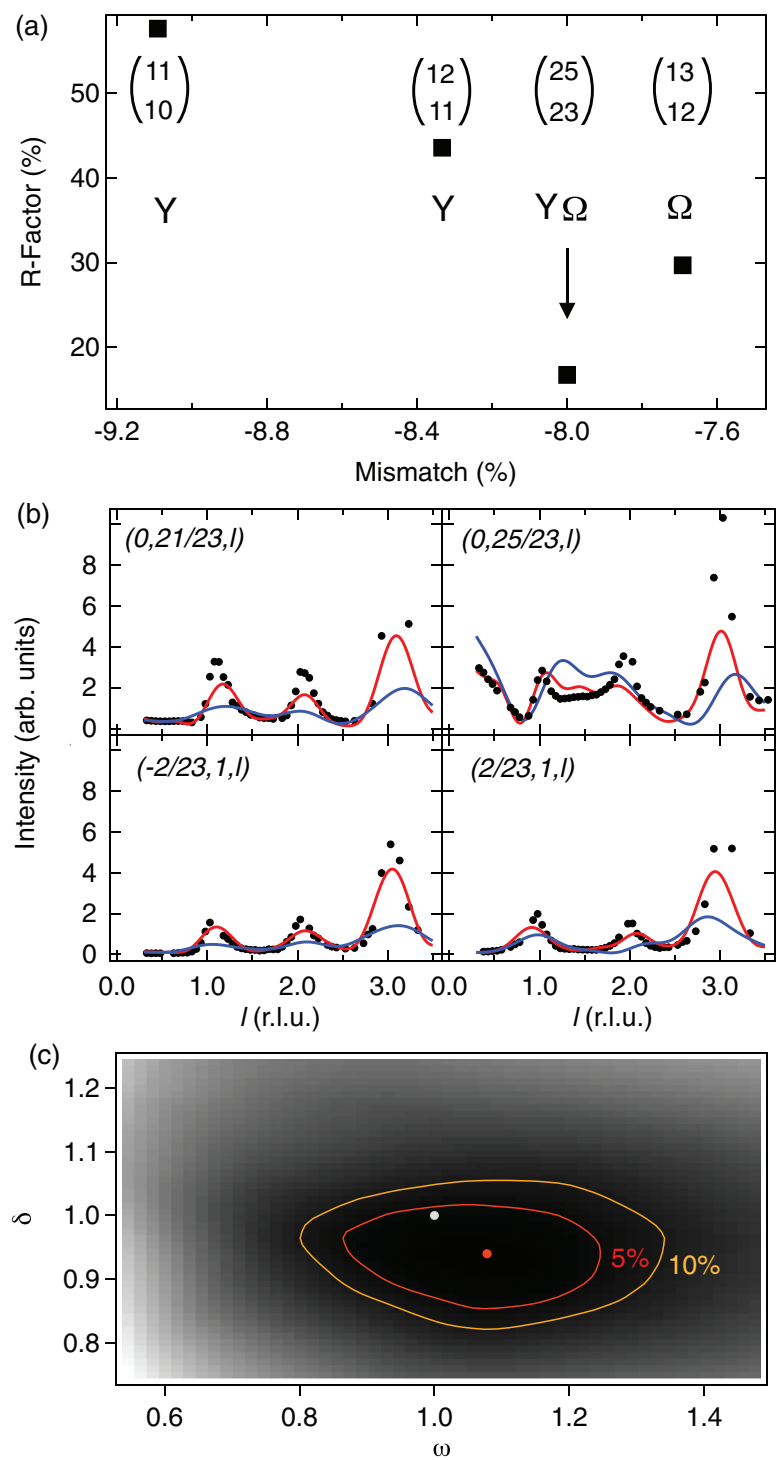

FIG. 3. (Color online) Comparison of SXRD data with DFT models of $g / \mathrm{Ru}(0001)$. (a) R-factors of four-Ru-layer models versus mismatch between the graphene and the substrate for models with $Y$ and $\Omega$ hills, respectively. (b) Illustration of the agreement between theory (solid lines) and experiment (black dots) for four superstructure rods of the $\left(\begin{array}{c}23 \\ 25\end{array}\right)$ structure. Blue: four-Ru-layer model; red: six-Rulayer model. (c) Two-parameter R-factor map. $\omega$ is a strain parameter, $\delta$ describes the shortest C-Ru distance. The six-layer $\left(\begin{array}{l}25 \\ 23\end{array}\right)$ DFT result $(\omega=1, \delta=1)$ lies within the $1.05 \mathrm{R}_{\min }$ contour.

in the $\left(\begin{array}{c}25 \\ 23\end{array}\right)$. This clearly demonstrates that SXRD is able to distinguish between different moirons, where we note that the footprint in the Ru substrate is important for the magnitude of this effect, because of the much stronger scattering power of $\mathrm{Ru}$ as compared to carbon.

In Fig. 3(b), the data from four superstructure rods are compared with the four-layer and six-layer models. The improvement of the agreement by including more substrate layers is obvious.

In order to further test the $\left({ }_{23}^{25}\right)$ DFT result with SXRD, we parametrized the DFT coordinates with a linear strain factor 
$\omega$, where $\omega=1$ corresponds to the $\left({ }_{23}^{25}\right)$ DFT coordinates and $\omega=0$ to flat graphene on flat ruthenium. The use of this parameter is motivated by the fact that the strain in the graphene is proportional to the strain in the substrate. To include the effect of the $\mathrm{C}-\mathrm{Ru}$ bond length, a second parameter $\delta$ was introduced, where $\delta=1$ corresponds to the smallest vertical C-Ru distance of $2.06 \AA$ from DFT.

In Fig. 3(c) the corresponding comparison between DFT coordinates and experimental data, i.e., the R-factor map is shown. The R-factor minimum lies very close to the predictions of the DFT model. The $1.05 \mathrm{R}_{\min }$ contour line is considered to be a fair limit for parameters that are consistent with experiment. The best fit yields $\omega_{\min }=1.02 \pm 0.21$ and $\delta_{\min }=0.96 \pm 0.08$. The confidence intervals are the halfwidth of the corresponding $\mathrm{R}$-factor parabola at $R=1.05$ $R_{\min }$. Both parameter ranges (or confidence intervals) contain the theory values of 1.0. Hence, experiment agrees with the theoretical prediction. From the parameter ranges, it can be seen that SXRD is more sensitive to $\delta$ than to $\omega$. The present $\delta$ values make C-Ru distance values below $1.8 \AA$ (Refs. 27 and 28) unlikely. The $\omega$ values indicate a graphene corrugation which favors none of the reported values. ${ }^{21,23,29}$ The result from the first SXRD model reported a corrugation of $0.82 \pm$ $0.15 \AA . .^{21}$ This was smaller than the value from the low-energy electron-diffraction (LEED) study of $1.5 \pm 0.2 \AA,{ }^{29}$ and larger than the value of $0.2 \AA$ derived from $\mathrm{He}$-scattering data. ${ }^{23}$ If we do not want to rely on the $\omega$ parameter for the determination of the graphene corrugation, because a change of the substrate corrugation affects the R-factor more strongly than does a proportional change of the graphene corrugation, we have to investigate a parametrization $\gamma$ that varies the graphene corrugation alone. Of course, a value of $\gamma=0$ is unphysical, because uncorrugated graphene will impose no strain in the substrate. However, if $\gamma=1$ corresponds to the corrugation of the DFT result, we obtain $\gamma_{\min }=0.8 \pm 0.4$. Therefore the graphene corrugation of the present DFT result is consistent with SXRD, although within a relatively large confidence interval.

In summary, the structure of the $\left(\begin{array}{c}25 \\ 23\end{array}\right)$ unit cell of graphene on ruthenium, of a large-scale density functional theory calculation, is presented. As confirmed by scanning tunneling microscopy and surface $\mathrm{x}$-ray diffraction, the unit cell contains two different moiré beatings that do not differ in corrugation height but have differently coordinated peaks and footprints in the substrate. These findings are important for the exploitation of large superstructures as templates with new functionalities.

Financial support from the Swiss National Science Foundation, computational resources provided by the Swiss Center for Computational Science, and the Sino-Swiss Science and Technology program are gratefully acknowledged. This work was partly performed at the Swiss Light Source, Paul Scherrer Institut. *greber@physik.uzh.ch

${ }^{1}$ M. Batzill, Surf. Sci. Rep. 67, 83 (2012).

${ }^{2}$ T. Brugger, S. Günther, B. Wang, J. H. Dil, M.-L. Bocquet, J. Osterwalder, J. Wintterlin, and T. Greber, Phys. Rev. B 79, 045407 (2009).

${ }^{3}$ I. Pletikosić, M. Kralj, P. Pervan, R. Brako, J. Coraux, A. T. N'Diaye, C. Busse, and T. Michely, Phys. Rev. Lett. 102, 056808 (2009).

${ }^{4}$ P. Sutter, M. S. Hybertsen, J. T. Sadowski, and E. Sutter, Nano Lett. 9, 2654 (2009).

${ }^{5}$ A. T. N'Diaye, S. Bleikamp, P. J. Feibelman, and T. Michely, Phys. Rev. Lett. 97, 215501 (2006).

${ }^{6}$ A. J. Pollard, E. W. Perkins, N. A. Smith, A. Saywell, G. Goretzki, A. G. Phillips, S. P. Argent, H. Sachdev, F. Mueller, S. Huefner, S. Gsell, M. Fischer, M. Schreck, J. Osterwalder, T. Greber, S. Berner, N. R. Champness, and P. H. Beton, Angew. Chem., Int. Edit. 49, 1794 (2010).

${ }^{7}$ H. T. Zhou, J. H. Mao, G. Li, Y. L. Wang, X. L. Feng, S. X. Du, K. Muellen, and H. J. Gao, Appl. Phys. Lett. 99, 153101 (2011).

${ }^{8}$ H. Zhou, L. Zhang, J. Mao, G. Li, Y. Zhang, Y. Wang, S. Du, W. A. Hofer, and H.-J. Gao, Nano Res. 6, 131 (2013).

${ }^{9}$ A. Zangwill, Physics at Surfaces, 1st ed. (Cambridge University Press, Cambridge, 1988).

${ }^{10}$ L. Gao, J. R. Guest, and N. P. Guisinger, Nano Lett. 10, 3512 (2010).

${ }^{11}$ Y. Pan, D.-X. Shi, and H.-J. Gao, Chin. Phys. 16, 3151 (2007).

${ }^{12}$ S. Marchini, S. Günther, and J. Wintterlin, Phys. Rev. B 76, 075429 (2007).

${ }^{13}$ A. L. Vázquez de Parga, F. Calleja, B. Borca, M. C. G. Passeggi, Jr., J. J. Hinarejos, F. Guinea, and R. Miranda, Phys. Rev. Lett. 100, 056807 (2008).
${ }^{14}$ D. Martoccia, P. R. Willmott, T. Brugger, M. Björck, S. Günther, C. M. Schlepütz, A. Cervellino, S. A. Pauli, B. D. Patterson, S. Marchini, J. Wintterlin, W. Moritz, and T. Greber, Phys. Rev. Lett. 101, 126102 (2008).

${ }^{15}$ K. Hermann, J. Phys.: Condens. Matter 24, 314210 (2012).

${ }^{16}$ B. Wang, M.-L. Bocquet, S. Marchini, S. Guenther, and J. Wintterlin, Phys. Chem. Chem. Phys. 10, 3530 (2008).

${ }^{17}$ B. Wang, S. Guenther, J. Wintterlin, and M.-L. Bocquet, New J. Phys. 12, 043041 (2010).

${ }^{18}$ M. Iannuzzi and J. Hutter, Surf. Sci. 605, 1360 (2011).

${ }^{19}$ D. Stradi, S. Barja, C. Diaz, M. Garnica, B. Borca, J. J. Hinarejos, D. Sanchez-Portal, M. Alcami, A. Arnau, A. L. Vazquez de Parga, R. Miranda, and F. Martin, Phys. Rev. Lett. 106, 186102 (2011).

${ }^{20} \mathrm{CP} 2 \mathrm{~K}$ version 2.2.1 (development version), the $\mathrm{CP} 2 \mathrm{~K}$ Developers Group (2012). cp2k is freely available under gpl license from http://www.cp2k.org.

${ }^{21}$ D. Martoccia, M. Bjoerck, C. M. Schlepuetz, T. Brugger, S. A. Pauli, B. D. Patterson, T. Greber, and P. R. Willmott, New J. Phys. 12, 043028 (2010)

${ }^{22}$ See Supplemental Material at http://link.aps.org/supplemental/ 10.1103/PhysRevB.88.125433 for the description of calculational and experimetal details, calculated graphene densities of states, a table on the results on different unit cells, and details of the SXRD evaluation.

${ }^{23}$ B. Borca, S. Barja, M. Garnica, M. Minniti, A. Politano, J. M. Rodriguez-Garcia, J. J. Hinarejos, D. Farias, A. L. V. de Parga, and R. Miranda, New J. Phys. 12, 093018 (2010).

${ }^{24}$ K. L. Man and M. S. Altman, Phys. Rev. B 84, 235415 (2011). 
${ }^{25}$ C. Schleputz, R. Herger, P. Willmott, B. Patterson, O. Bunk, C. Bronnimann, B. Henrich, G. Hulsen, and E. Eikenberry, Acta Cryst. A 61, 418 (2005).

${ }^{26}$ M. Bjoerck, J. Appl. Crystallogr. 44, 1198 (2011).

${ }^{27}$ P. W. Sutter, J.-I. Flege, and E. A. Sutter, Nat. Mater. 7, 406 (2008).
${ }^{28}$ E. Sutter, P. Albrecht, B. Wang, M.-L. Bocquet, L. Wu, Y. Zhu, and P. Sutter, Surf. Sci. 605, 1676 (2011).

${ }^{29}$ W. Moritz, B. Wang, M.-L. Bocquet, T. Brugger, T. Greber, J. Wintterlin, and S. Günther, Phys. Rev. Lett. 104, 136102 (2010). 